\title{
Human motion capturing system with MEMS accelerometers (notice of removal)
}

\section{Baoping Xiao, Chang Xu, Lijun Xu, Shuigeng Ouyang}

Baoping Xiao, Chang Xu, Lijun Xu, Shuigeng Ouyang, "Human motion capturing system with MEMS accelerometers (notice of removal)," Proc. SPIE 6724, 3rd International Symposium on Advanced Optical Manufacturing and Testing Technologies: Design, Manufacturing, and Testing of Micro- and Nano-Optical Devices and Systems, 672413 (21 November 2007); doi: $10.1117 / 12.782829$ 


\title{
Human motion capturing system with MEMS accelerometers (notice of removal)
}

\author{
Proc. SPIE 6724, 672413 (2007); http://dx.doi.org/10.1117/12.782829
}

Online Publication Date: 14 November 2007

Retracted from Publication: 12 April 2010

Conference Date: 12 July 2007

Conference Location: Chengdu, China

Conference Title: 3rd International Symposium on Advanced Optical Manufacturing and Testing

Technologies: Design, Manufacturing, and Testing of Micro- and Nano-Optical Devices and Systems

Conference Chairs: Sen Han, Tingwen Xing, Yanqiu Li, Zheng Cui

\section{Baoping Xiao}

China Jiliang Univ. (China) and Zhejiang Univ (China)

Chang Xu, Lijun Xu, and Shuigeng Ouyang

China Jiliang Univ. (China)

This paper (672413) was removed from the SPIE Digital Library on 13 April 2010 to discovery of plagiarism. As stated in the SPIE Guidelines for Professional Conduct and Publishing EthicS, SPIE defines plagiarism as the reuse of someone else's prior ideas, processes, results, or words without explicit attribution of the original author and source, or falsely representing someone else's work as one's own. SPIE considers plagiarism in any form, at any level, to be unacceptable and a serious breach of professional conduct. It is SPIE policy to remove such papers and to take appropriate corrective or disciplinary action against the offending author(s). 